\title{
Guest Editorial: Global modeling using local patterns
}

\author{
Johannes Fürnkranz • Arno Knobbe
}

Published online: 11 April 2010

The Author(s) 2010. This article is published with open access at Springerlink.com

\section{Introduction}

Over the last decade, local pattern discovery has become a rapidly growing field (Morik et al. 2005), and a range of techniques is available for producing extensive collections of patterns. Because of the exhaustive nature of most such techniques, the pattern collections provide a fairly complete picture of the information content of the database. However, such so-called local patterns represent fragmented knowledge, and it is often not clear how the pieces of the puzzle can be combined into a global model, which is often the desirable result of a data mining process. Thus, the question of how to turn large collections of patterns into global models deserves attention.

This special issue of the Data Mining and Knowledge Discovery Journal features a number of papers that represent the state of the art in building global models from local patterns. In our view, a common ground of all the local pattern mining techniques is that they can be considered to be feature construction techniques that follow different objectives (or constraints). We will see that the redundancy of these patterns and the selection of suitable subsets of patterns are addressed in separate steps, so that each resulting feature is highly informative in the context of the global data mining problem.

In earlier work (Knobbe et al. 2008), a framework was proposed that provides a general outline of the activities involved. The framework, called From Local Patterns

\footnotetext{
J. Fürnkranz ( $\varangle)$

TU Darmstadt, Darmstadt, Germany

e-mail: juffi@ke.tu-darmstadt.de
}

A. Knobbe

LIACS, Leiden University, Leiden, The Netherlands

e-mail: knobbe@liacs.nl 
to Global Models (LeGo), consists of a number of steps. Each step can be implemented by a range of techniques from the literature, making the framework general and of value to practitioners wishing to apply their favorite algorithm in a wider context. Furthermore, it subsumes a number of existing global methods based on pattern discovery. The framework helps analyzing and improving such methods by relating it to other similar methods, and suggesting alternative options for individual steps in the process. The papers that feature in this special issue all describe approaches that, to various degrees, fit the general model.

\section{The LeGo framework}

The LeGo framework can be thought of as a refinement of the conventional KDD process model, as it has been sketched in similar ways in numerous publications, going back as far as 1996 (Fayyad et al. 1996):

1. Feature construction Essentially, the process starts with a data source (typically a relational database) that needs to be prepared for the mining process. The first phase, known as feature construction, produces from the initial data source a so-called feature base, by means of some, typically manual or semi-automatic, transformation process. The purpose of this transformation may be to extract specific, potentially useful information that is only represented implicitly in the data source (e.g. translating purchase-date into a weekend/weekday indicator).

2. Feature selection Once the data source is transformed into a feature base, the feature selection phase (Guyon and Elisseeff 2003) is responsible for selecting a subset of these features (the mining base). This is particularly important when large numbers of features are generated. Typical problems with large feature spaces include text mining (Forman 2003), propositionalization approaches to relational learning (Kramer et al. 2001), and others.

3. Model construction Finally, a model construction phase involves applying one of the many available inductive methods to produce a model from the mining base. In descriptive data mining, the model itself is of primary interest. In predictive data mining, the model is used for making predictions, basically treating it as a black box.

The LeGo framework can be viewed as an instance of this general process model, with local patterns, rather than features, being the prime subject. Local patterns informally refers to regularities that hold for a particular part of the data. The term local indicates that it captures some aspect of the data, without providing a complete picture of the database. Local patterns do not necessarily represent exceptions in the data (Hand 2002), but rather fragmented and incomplete knowledge, which may still be fairly general.

As shown in Fig. 1, the framework is centered around three important phases, which correspond to the three phases mentioned above: 


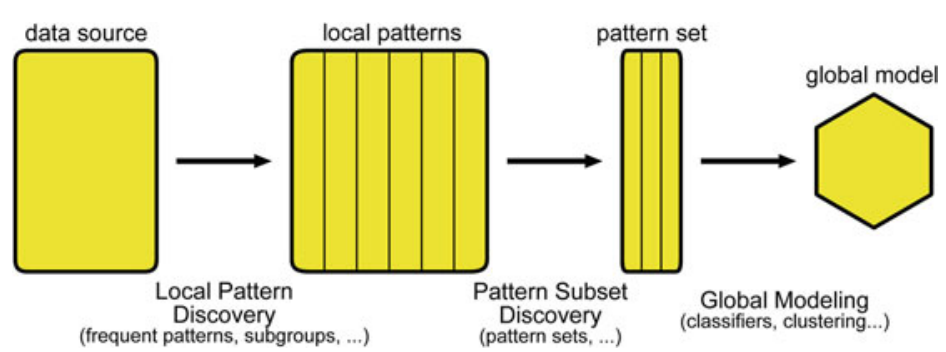

Fig. 1 The LeGo framework (Knobbe et al. 2008)

\section{Local pattern discovery}

2. Pattern Subset discovery

3. Global modeling

These three phases are explained in detail in the following sections.

\subsection{Local pattern discovery}

The local pattern discovery phase is responsible for producing a set of candidate patterns by means of an exploratory analysis of a search-space of patterns, defined by a set of inductive constraints provided by the user. As such, this phase can be seen as an automated instance of the feature construction phase in the KDD process. Patterns are typically judged on qualities such as their frequency or predictive power with respect to some target concept.

The most basic, and at the same time most popular type of local pattern discovery is the (unsupervised) discovery of frequent itemsets (Goethals 2005). Clearly, a frequent itemset is an incomplete representation of some aspect of the distribution of items, and of possible co-occurrences among the items (associations). An itemset is local because it covers only the part of the database for which its items are supported.

Another important example of local pattern discovery is known as Subgroup Discovery, sometimes referred to as Correlated Pattern Discovery (Kralj Novak et al. 2009; Zimmermann and DeRaedt 2009). The main goal is to identify patterns that are interesting, in the sense that they are well supported and that the set of covered examples differs substantially from the overall population with respect to the distribution of its boolean (or nominal) target attribute.

These two prototypical discovery methods demonstrate an important concept: local patterns can be interpreted as features, in this case binary features. The set of conditions represented by the pattern (subgroup, frequent itemset, ...) either does or does not hold for a given example. Thus, any data mining operation that works on binary features can be employed in the subsequent phases of Pattern Subset Discovery and Global Modeling. In many cases, these subsequent operations will simply ignore any information concerning the structure of the patterns, and will focus only on the resulting feature. This interpretation emphasizes the locality of patterns: each pattern helps to identify some important subset of the database that exhibits some properties that distinguish it from its complement. 


\subsection{Pattern subset discovery}

This phase considers the potentially large collection of patterns produced in the preceding phase, and selects from those a compact set of informative and relevant patterns that shows little redundancy. This phase is the counterpart of the feature selection phase in the KDD process.

In the local pattern discovery phase, patterns are discovered on the basis of their individual merits. In practice, this results in large sets of local patterns, with potentially high levels of redundancy among the patterns. For manual inspection of the collection of patterns, reporting more than a handful of patterns is clearly infeasible. Furthermore, when inducing global models from the set of local patterns, machine learning procedures tend to be hindered by the presence of many, often redundant, features. The goal of the Pattern Subset Discovery phase therefore, is to reduce the redundancy by selecting a subset of patterns from the initial large set on the basis of their usefulness in the context of other patterns selected.

Several approaches have been proposed to reduce the number of local patterns irrespective of their subsequent use. Recently, two approaches to Pattern Subset Discovery have appeared in the literature, which explicitly represent the goal of combining and selecting patterns: constraint-based pattern set mining (De Raedt et al. 2007), and pattern teams (Knobbe and Ho 2006a, b). In broad terms, these approaches are very similar. Both assume that the syntactic structure of the individual patterns is irrelevant at this stage, and that patterns can be fully characterized by a binary feature that determines for each example whether it is covered by the pattern or not.

\subsection{Global modeling}

This phase is responsible for turning the condensed set of relevant patterns into a well-balanced global model. The global modeling phase either treats each local pattern as a constructed feature, and applies an existing inductive method, or applies some pattern combination strategy that is specific to the class of patterns discovered.

Computing a global model from a set of local patterns (i.e. features) can be quite straightforward; we may basically utilize any machine learning algorithm at this point, most of which will clearly benefit from high quality features. It is well known that good features often contribute more to data mining success than the selection of a specific algorithm and the fine-tuning of its parameters. Although this generic use of learning techniques for global modeling is clearly advocated in the LeGo framework, up to recently many approaches employed fairly ad hoc techniques, or used methods that depend on the specific nature of the local patterns (e.g. itemsets or clusters).

Especially in the discipline of (association) rule discovery, the problem of building a unified global classification model has been approached by so-called combination strategies, an idea that goes back to the concept of ensembles (or multiple classifier systems). If we consider each local pattern as a weak classifier, we can (arguably) construct our global model as an ensemble of patterns. We now have a range of 
proposed combination strategies at our disposal that effectively assign a weight to each rule or pattern (Zimmermann and DeRaedt 2004).

This evolution of strategies naturally leads to the final approach of applying arbitrary learning methods to combine sets of patterns, assuming again that every pattern can be interpreted as a (binary) feature. The use of generic induction methods is advocated for example in the recent correlated pattern mining approach (Bringmann et al. 2006) and the Safarii system (Knobbe 2006).

\section{Benefits of building global models from local patterns}

As building global models is clearly still an important goal, one might wonder why such a global model cannot be induced directly, as is customary in traditional inductive methods. Why spend the extra time to search for an exhaustive collection of patterns, if most of them are later discarded due to redundancy or irrelevancy?

A key motivation comes from the expected accuracy gains resulting from the more exploratory or exhaustive nature of the initial pattern discovery step. Many successful machine learning techniques implicitly include an automated pattern discovery phase. For example, the nodes in the hidden layer of a multi-layer perceptron will typically converge to several useful subconcepts, which may be important for some (but not necessarily all) of the output nodes. Similarly, kernel methods perform an implicit feature generation step. On the other hand, there are also widely used pre-processing techniques like principal components analysis that perform feature generation with the goal of supporting a subsequent modeling step. Further examples include text mining techniques like probabilistic latent semantic analysis (Hofmann 1999) and latent Dirichlet allocation (Blei et al. 2003), which introduce a number of latent topics that serve as an intermediate semantic layer capturing important regularities in the input space. For all these methods, the intermediate layer allows to abstract the input features into more meaningful or more discriminative local patterns.

Thus, it seems to be a good idea to adopt local pattern discovery techniques as a pre-processing step for global modeling. In practice, globally useful features can usually be assumed to also perform locally well to a certain degree, which means that they can be detected by local pattern mining techniques. Moreover, the set of discovered patterns is typically complete (within the inductive constraints), which means that in subsequent phases, two patterns of moderate quality could be combined to form a perfect model. Global modeling algorithms, on the other hand, often tend to be greedy, and are likely to miss combinations of complex patterns (think of the XOR-problem).

Another key advantage of this approach is that the local pattern discovery can, to some extent, be performed independently of subsequent global modeling steps. The found patterns can be stored as an intermediate result that could be put to use for a variety of different global modeling tasks. Depending on the concrete implementation of the LeGo framework, one can store individual patterns or entire pattern sets. One should, however, keep in mind that in some cases it could be desirable to tailor the patterns or pattern sets to the concrete global modeling task. It is an open research problem, how constraints from the global task can be propagated back to the local pattern discovery phase. 
Finally, although we are promoting the construction of global models from local patterns, the global models themselves may not necessarily be the desired end-product. The local patterns could still be the prime subject, and global modeling could serve as a means of validating candidate pattern sets. One could argue that a pattern team (Knobbe and Ho 2006a) that optimizes a certain classifier represents a set of patterns that are worth inspecting manually, as relevancy and lack of redundancy are mostly guaranteed. Additionally, going back from the pattern subset discovery phase to the local pattern discovery phase, it makes sense to see what patterns were accepted into a pattern team, and how they relate to the remaining patterns. Potentially, pattern team members may be replaced by alternative patterns with identical binary features. It is interesting to see why such patterns are the same or similar, syntactically or semantically.

\section{The special issue}

The papers collected in this special issue tackle some of the above questions, highlighting on-going work in this area.

In the first paper, Nijssen and Fromont (2010) demonstrate how frequent itemsets can be used for constructing optimal decision trees under a large variety of constraints. The paper provides a systematic discussion of different types of constraints, and proposes an approach for generating decision trees from lattices of itemsets that have been mined under these constraints. Two versions of the algorithm are able to mine decision trees from an existing lattice, or to integrate the mining phase into the learning algorithm. Because of the itemset mining component, the algorithm has some practical limitations for example in terms of the number of attributes and their types or in terms of the constraints that should be specified by the user. It has however considerable theoretical interest.

Dembczynski et al. (2010) describe ENDER, a general statistical framework for boosting decision rules. This work is particularly interesting because it demonstrates how different loss functions for the performance of the global model can be decomposed into impurity measures that can be optimized during the construction of local patterns in the form of individual classification or regression rules. The paper evaluates the approach for various different loss functions and minimization techniques. It also compares a wide variety of boosting-based rule learning algorithms, like Slipper (Cohen and Singer 1999), RuleFit (Friedman et al. 2008), LRI (Weiss and Indurkhya 2000), and ENDER, which by itself is already a valuable contribution in this field.

Azevedo et al. (2010) introduce and evaluate two alternative approaches to classification by association. While conventional approaches, such as CBA (Liu et al. 1998), directly combine the found local patterns into a single global rule base, the approaches introduced here form ensemble classifiers from the found rules. The first approach, Post Bagging, takes different random samples of rules from a large base of found rules. The resulting rule sets are then combined as in conventional rule-based bagging. The second approach, Iterative Reordering Ensembling (IRE), forms an ensemble of different orderings of the rules in a decision list, whose predictions are, again, combined at classification time. In an extensive set of experiments on 36 datasets, the IRE approach 
performed somewhat better than Post Bagging, and compared well with alternative rule-based ensemble methods.

Wiswedel et al. (2010) introduce learning from parallel universes as a framework for learning from multiple representations of instances. The key idea is that patterns that are local in one of the different representation spaces are combined into a model which is global across all representations. This differs from previous approaches to multi-view learning, which typically learn models that are global in all different views. The approach is illustrated on an extension of conventional fuzzy and probabilistic clustering algorithms to the used multiple representations.

The key idea of Malik et al. (2010) is to mine local patterns of length two in order to employ them for a hierarchical document clustering task. The patterns are evaluated with a trade-off between their local frequency in the document and their global frequency in all documents. The authors emphasize that a separate mining of local patterns in each document is crucial for the good performance of their algorithm, which outperforms (on average) alternative state-of-the-art clustering methods on 16 standard text classification sets, and for automated construction of descriptive cluster labels as an integral part of the clustering process.

Finally, Jaroszewicz (2010) describes the use of local patterns in the form of event sequences for the interactive construction of a global Hidden Markov Model. The local pattern discovery focuses on sequences that occur more frequently than it would be expected from the constructed model. Discovered patterns are then given to the analyst who manually updates the model using their understanding of the domain. Thus, phases of local and global mining can succeed each other iteratively. The author shows that such an interactive construction may yield better results than a fully automated approach in applications like Web log mining and protein secondary structure prediction.

Acknowledgements We would like to thank all authors who submitted their work to this special issue (in particular to those whose fine works did eventually not make the cut) and our reviewers, whose careful comments on the submitted papers contributed to this final selection of papers. Special thanks go to Bruno Crémilleux and Martin Scholz for their contributions to the LeGo framework. This work has been supported by the German Science Foundation (DFG).

Open Access This article is distributed under the terms of the Creative Commons Attribution Noncommercial License which permits any noncommercial use, distribution, and reproduction in any medium, provided the original author(s) and source are credited.

\section{References}

Azevedo PJ, Jorge AM (2010) Ensembles of jittered association rule classifiers. Data Min Knowl Disc. doi:10.1007/s10618-010-0173-y

Blei DM, Ng AY, Jordan MI (2003) Latent dirichlet allocation. J Mach Learn Res 3:993-1022

Bringmann B, Zimmermann A, De Raedt L, Nijssen S (2006) Don't be afraid of simpler patterns. In: Fürnkranz J, Scheffer T, Spiliopoulou M (eds) Proceedings of the 10th European conference on principles and practice of knowledge discovery in databases (PKDD-06). Springer-Verlag, Berlin, Germany, pp 55-66

Cohen WW, Singer Y (1999) A simple, fast, and effective rule learner. In: Proceedings of the 16th national conference on artificial intelligence (AAAI-99). AAAI/MIT Press, Menlo Park, CA, pp 335-342 
De Raedt L, Zimmermann A (2007) Constraint-based pattern set mining. In: Proceedings of the 7th SIAM international conference on data mining (SDM-07), Bethesda, MD

Dembczynski K, Kotłowski W, Słowinski R (2010) ENDER—a statistical framework for boosting decision rules. Data Min Knowl Disc. doi:10.1007/s10618-010-0177-7

Fayyad UM, Piatetsky-Shapiro G, Smyth P (1996) From data mining to knowledge discovery in databases. AI Mag 17(3):37-54

Forman G (2003) An extensive empirical study for feature selection in text classification. J Mach Learn Res 3:1289-1305

Friedman JH, Popescu BE (2008) Predictive learning via rule ensembles. Ann Appl Stat 2:916-954

Giacometti A, Khanjari ME, Marcel P, Soulet A (2009) A framework for pattern-based global models. In: Proceedings of the 10th international conference on intelligent data engineering and automated learning. Burgos, Spain

Goethals B (2005) Frequent set mining. In: Maimon O, Rokach L (eds) The data mining and knowledge discovery handbook. Springer-Verlag, Berlin, pp 377-397

Guyon I, Elisseeff A (2003) An introduction to variable and feature selection. J Mach Learn Res 3:11571182

Hand DJ (2002) Pattern detection and discovery. In: Hand DJ, Adams NM, Bolton RJ (eds) Proceedings of the ESF exploratory workshop on pattern detection and discovery in data Mining. Springer, pp 1-12

Hofmann T (1999) Probabilistic latent semantic analysis. In: Proceedings of the 15 th conference on uncertainty in artificial intelligence (UAI-99). Stockholm, Sweden, pp 289-296

Jaroszewicz S (2010) Using interesting sequences to interactively build Hidden Markov Models. Data Min Knowl Disc. doi:10.1007/s10618-010-0171-0

Knobbe AJ (2006) Safarii multi-relational data mining environment. http://www.kiminkii.com/safarii.html

Knobbe AJ, Crémilleux B, Fürnkranz J, Scholz M (2008) From local patterns to global models: the LeGo approach to data mining. In: Fürnkranz J, Knobbe A (eds) From local patterns to global models. Proceedings of the ECML PKDD 2008 Workshop. Antwerp, Belgium, pp 1-16. http://www.ke.informatik. tu-darmstadt.de/events/LeGo-09/

Knobbe AJ, Ho EKY (2006) Maximally informative $k$-itemsets and their efficient discovery. In: Eliassi-Rad T, Ungar LH, Craven M, Gunopulos D (eds) Proceedings of the 12th ACM SIGKDD international conference on knowledge discovery and data mining (KDD-06). Philadelphia, PA, pp 237-244

Knobbe AJ, Ho EKY (2006) Pattern teams. In: Fürnkranz J, Scheffer T, Spiliopoulou M (eds) Proceedings of the 10th European conference on principles and practice of knowledge discovery in databases (PKDD-06). Springer-Verlag, Berlin, Germany, pp 577-584

Kralj Novak P, Lavrač N, Webb GI (2009) Supervised descriptive rule discovery: a unifying survey of contrast set emerging pattern and subgroup mining. J Mach Learn Res 10:377-403

Kramer S, Lavrač N, Flach P (2001) Propositionalization approaches to relational data mining. In: Džeroski S, Lavrač N (eds) Relational data mining. Springer-Verlag, Berlin, pp 262-291

Liu B, Hsu W, Ma Y (1998) Integrating classification and association rule mining. In: Agrawal R, Stolorz P, Piatetsky-Shapiro G (eds) Proceedings of the 4th international conference on knowledge discovery and data mining (KDD-98), pp 80-86

Malik HH, Kender JR, Fradkin D, Moerchen F (2010) Hierarchical document clustering using local patterns. Data Min Knowl Disc. doi:10.1007/s10618-010-0172-z

Morik K, Boulicaut J-F, Siebes A (eds) (2005) Local pattern detection. Springer-Verlag, Berlin

Nijssen S, Fromont E (2010) Optimal constraint-based decision tree induction from itemset lattices. Data Min Knowl Disc. doi:10.1007/s10618-010-0174-x

Weiss SM, Indurkhya N (2000) Lightweight rule induction. In: Langley P (eds) Proceedings of the 17th international conference on machine learning (ICML-2000). Stanford, CA, pp 1135-1142

Wiswedel B, Höppner F, Berthold MR (2010) Learning in parallel universes. Data Min Knowl Disc. doi:10. 1007/s10618-010-0170-1

Zimmermann A, DeRaedt L (2004) Corclass: correlated association rule mining for classification. In: Suzuki E, Arikawa S (eds) Proceedings of the 7th international conference on discovery science (DS'04). Springer-Verlag, Berlin, pp 60-72

Zimmermann A, DeRaedt L (2009) Cluster-grouping: from subgroup discovery to clustering. Mach Learn 77(1):125-159 